\title{
Thioesters Provide a Robust Path to Prebiotic Peptides
}

\author{
Moran Frenkel-Pinter ${ }^{\mathrm{a}, \mathrm{b}}$, Marcos Bouza ${ }^{\mathrm{a}, \mathrm{b}}$, Facundo M. Fernández ${ }^{\mathrm{a}, \mathrm{b}}$, Luke J. Leman ${ }^{\mathrm{a}, \mathrm{c}}$, Loren \\ Dean Williams $^{\mathrm{a}, \mathrm{b}}$, Nicholas V. Hud ${ }^{\mathrm{a}, \mathrm{b}^{*}}$, and Aikomari Guzman-Martinez ${ }^{\mathrm{a}, \mathrm{d}^{*}}$ \\ ${ }^{a}$ NSF-NASA Center for Chemical Evolution, Georgia Institute of Technology, Atlanta, GA 30332 (USA) \\ ${ }^{\mathrm{b}}$ School of Chemistry \& Biochemistry, Georgia Institute of Technology, Atlanta, GA 30332 (USA) \\ ${ }^{c}$ Department of Chemistry, The Scripps Research Institute, La Jolla, CA 92037 (USA) \\ ${ }^{d}$ Department of Chemistry, University of Puerto Rico, Mayagüez, Mayagüez, PR 00681 (USA) \\ *Co-corresponding authors \\ *Corresponding Authors: \\ Prof. Aikomari Guzman-Martinez \\ Department of Chemistry \\ University of Puerto Rico, Mayagüez \\ Mayagüez, PR 00681 \\ Ph: (+1) (787) 832-4040 \\ Fax: (+1) (787) 265-3849 \\ aikomari.guzman@upr.edu
}

Prof. Nicholas V. Hud

School of Chemistry and Biochemistry

Georgia Institute of Technology

315 Ferst Drive NW

Atlanta, GA 30332-0400

Ph: (+1) (404) 385-1162

Fax: (+1) (404) 894-2295

nick.hud@chemistry.gatech.edu 


\begin{abstract}
The condensation of building blocks into oligomers and polymers was an early and important stage in the origins of life. High activation energies, unfavorable thermodynamics and side reactions are bottlenecks for abiotic formation of peptides. Thioesters are hypothesized to have played key roles in prebiotic chemistry on early Earth, serving as energy storing molecules, as synthetic intermediates, and as catalysts in the formation of more complex molecules, including polypeptides. However, all abiotic reactions reported thus far for peptide formation via thioester intermediates have relied on activated building blocks or condensing agents, which are of questionable prebiotic relevance. We report robust, plausible prebiotic reactions of mercaptoacids with amino acids that result in the formation of peptides and thiodepsipeptides, which contain both peptide and thioester bonds. Peptide bond formation proceeds by the condensation of mercaptoacids to form thioesters followed by thioester-amide exchange. Mercaptoacids catalyze thiodepsipeptides and peptide formation under a wide range of $\mathrm{pH}$ conditions and at mild temperatures. Our results offer the most robust one-pot pathway for peptide formation ever reported. These results support the hypothesis that thiodepsipeptides formed robustly on prebiotic Earth and were possible contributors to early chemical evolution.
\end{abstract}

The condensation of building blocks into oligomers and polymers was an early and important stage in the origins of life. Thioesters are probable intermediates and catalysts during the origins of life, en route to the first complex oligomers and polymers. ${ }^{1-17}$ De Duve inferred an early role for thioesters based on their importance in modern metabolism ${ }^{18}$, where thioester intermediates enable the synthesis and degradation of peptides, fatty acids, sterols, and porphyrins. ${ }^{7,8}$ Moreover, thiols are thought to have been abundant on the prebiotic Earth, especially near sources of hydrogen sulfide. ${ }^{19}$

High activation energies, unfavorable thermodynamics and side reactions are bottlenecks for abiotic formation of peptides. Thioester intermediates might provide resolution, but thus far their formation has have required activated building blocks or condensing agents, which are prebiotically questionable. For example, thiodepsipeptides, which contain both peptide and thioester bonds, have been produced in various model prebiotic reactions from chemically activated building blocks. ${ }^{20-24}$ For example, peptides were formed via thioester intermediates in aqueous solution by Wieland and coworkers in 1953. These researchers synthesized valine-cysteine dipeptide through native chemical ligation, in which transthioesterification is followed by an intramolecular S,N-acyl shift, between cysteine and a valinethioester. ${ }^{20}$ Weber and Orgel later described thioester forming reactions using an N-Acyl protected cysteine or 3-mercaptopropionic acid. ${ }^{21-24}$ More recently, attempts to form peptides were made in aqueous solutions via oxidative acylation of thioacids. ${ }^{25-27}$ Amide bonds were also shown to form upon reductive acetylation 
of amino acids with mercaptoacetic acid in aqueous solutions, driven by the formation of pyrite $\left(\mathrm{FeS}_{2}\right)$ from FeS and $\mathrm{H}_{2} \mathrm{~S}^{28}$

We hypothesized a potentially simpler and more robust approach to prebiotic peptide bond formation in which dry-down reactions of mixtures of mercaptoacids and amino acids would reduce kinetic and thermodynamic barriers, and would produce linear thiodepsipeptide and peptide oligomers. It is known that a thiol and a carboxylic acid can condense to form a thioester (Fig. 1, top). These thioester linkages can then convert to peptide linkages via thioester-amide exchange (Fig. 1, bottom). This reaction scheme is analogous to the proposed acyl substitutions involved in the formation of depsipeptides, which contain mixtures of peptide and ester bonds. Specifically, amide bonds form via ester-amide exchange reactions in the presence of amino acids, which are enabled by the pre-formation of ester bonds between hydroxy acids under evaporative conditions. ${ }^{29-31}$ While depsipeptide formation is a promising prebiotically-relevant approach for formation of peptides on the abiotic Earth, it is limited to acidic conditions. We hereby report a much more robust prebiotic system for the formation of peptides and thiodepsipeptides by the reaction of mercaptoacids with amino acids. Compared to reactions involving hydroxy acids as catalysts, mercaptoacids catalyze thiodepsipeptide and peptide formation under a wider range of $\mathrm{pH}$ conditions and at much milder temperatures. This is explained by the higher nucleophilicity of thiols than alcohols as well as higher electrophilicity of thioesters than esters. ${ }^{32}$ Our results suggest that thiodepsipeptides could have formed robustly on the prebiotic Earth and were key players in the origins of life.

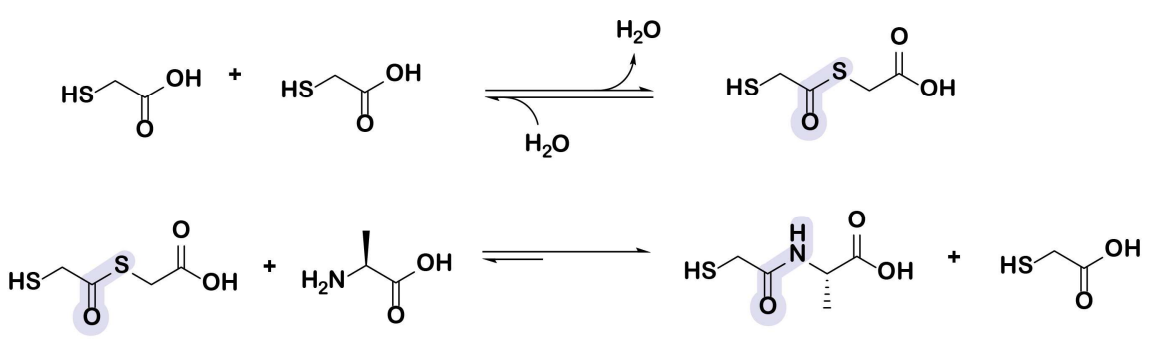

Fig. 1. Proposed acyl substitutions for peptide bond formation (amidation) through thioester-amide exchange. Mercaptoacids condense to form thioesters in dry conditions, which can be exchanged for amide bonds in the presence of amino acids.

\section{Results}

Thiodepsipeptides and peptides form via dry-down reactions of mercaptoacids and amino

acids. To test whether thiodepsipeptides form in dry-down reactions, we dried L-alanine (Ala) with thioglycolic acid (tg) for one week at $65{ }^{\circ} \mathrm{C}$ under unbuffered, mildly acidic conditions (ca. pH 3) (Fig. 2 ). Reactions were carried out in an anaerobic chamber to mimic early Earth conditions and to minimize thiol oxidation. Preliminary studies indicated oligomer formation with greater yields when the starting mixture contained an excess of mercaptoacid over amino acid (Supplementary Fig. 1). We therefore employed a 5:1 
mercaptoacid/amino acid molar ratio in subsequent reactions. Product mixtures for the reactions were characterized by a variety of analytical methods, including liquid chromatography mass spectrometry (LCMS), Fourier transform infrared spectroscopy (FTIR), high performance liquid chromatography (HPLC), and nuclear magnetic resonance (NMR).

Direct infusion MS and LC-MS analyses indicated that in dry-down reactions co-polymers of tg and Ala were formed (Fig. 2a). Mixtures of tg and Ala produced oligomers up to hexamers, with varying lengths and compositions of tg and Ala (Fig. 2a). Examples of thiodepsipeptide products include $3 \operatorname{tg} 3 \mathrm{Ala}$ and 1 tg $3 \mathrm{Ala}$, with each compositional species representing multiple possible sequences. Most oligomer products contained between one to three Ala units, and some observed species contained disulfide bonds (Fig. 2a and Supplementary Fig. 2). We confirmed the reproducibility of these results with independent replicate experiments (Supplementary Fig. 3).
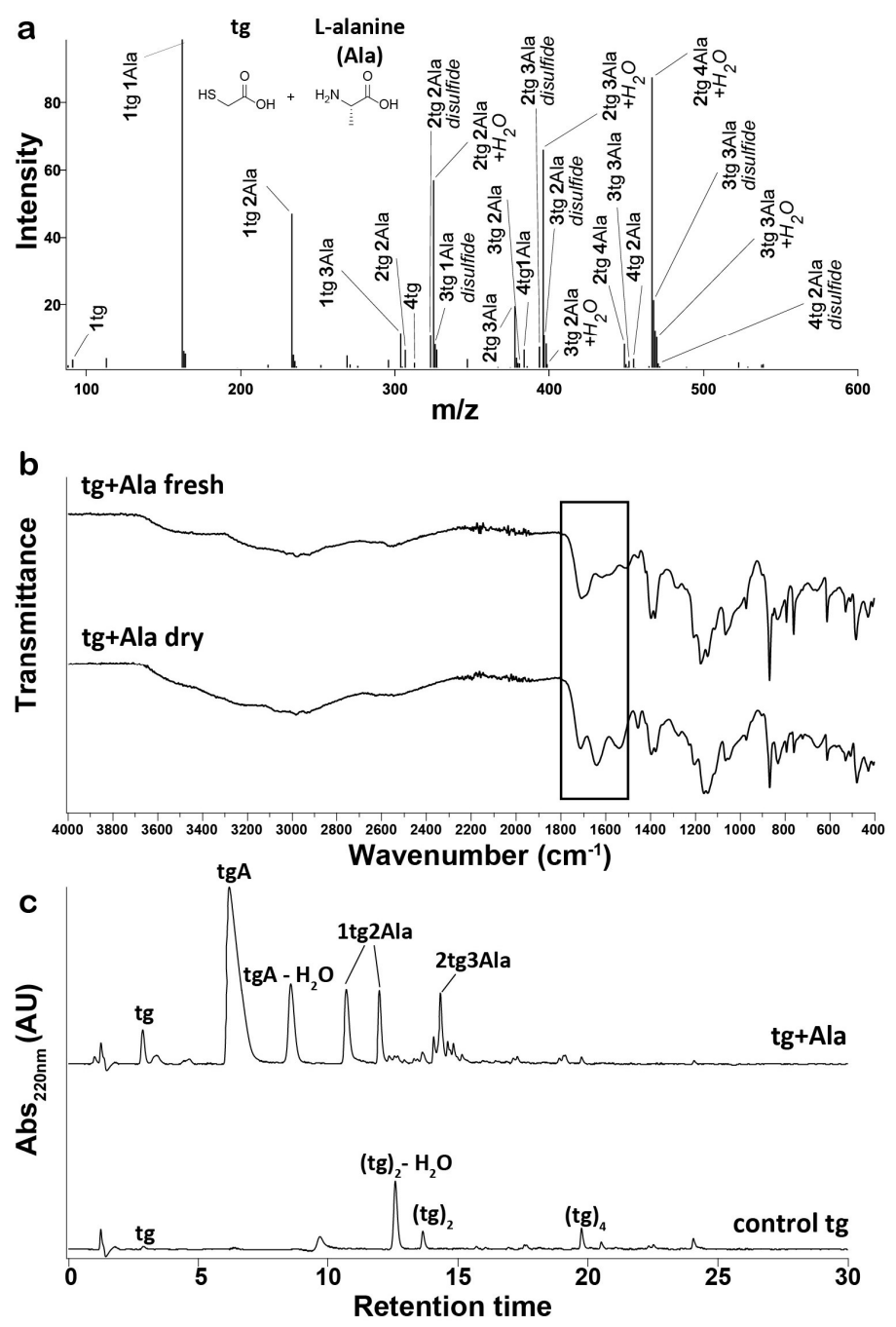

Fig. 2. Analytical data confirming formation of thiodepsipeptides in dry-down reactions of tg mixed with Ala. (a) Negative ion mode ESI-MS spectrum of tg and Ala after being dried at $65^{\circ} \mathrm{C}$ for seven days, indicating a variety of thiodepsipeptides. All labeled species correspond to $[\mathrm{M}-\mathrm{H}]^{-}$ions. Species that are 
labelled with $\mathrm{H}_{2} \mathrm{O}$ correspond to species that have an additional $\mathrm{H}_{2} \mathrm{O}$ unit, which could be a result of noncovalent adducts. (b) Fourier Transform Infrared Spectroscopy (FTIR) shows shifts in the $\mathrm{C}=\mathrm{O}$ band and in the amide regions upon dry-down of $\mathrm{tg}$ and Ala, thus supportive of thiodepsipeptide formation. (c) HPLC chromatograms of products resulting from drying tg alone or tg with Ala at $65{ }^{\circ} \mathrm{C}$ for seven days. Comparison between the two dry-down reaction products via hydrophobicity-based separation on a C18 column allows identification of various oligomers that contain Ala.

Verification of amide bonds within the product mixtures. The presence of amide bonds within the product oligomers was confirmed with FTIR (Fig. 2b). The dry-down reaction of tg and Ala resulted in the appearance of amide absorption bands (Amide I and Amide II), a direct report of amide bond formation $^{33}$, as well as changes in the thiol S-H stretch $\left(\sim 2570 \mathrm{~cm}^{-1}\right)^{34,35}$ and in the $\mathrm{C}=\mathrm{O}$ stretch (Fig. 2b). ${ }^{36-}$ ${ }^{38}$ Shifts in the $\mathrm{C}=\mathrm{O}$ stretch upon dry-down of a control reaction of tg alone, in the absence of amino acids, support the formation of thioesters by tg (Supplementary Fig. 4). ${ }^{36-38}$ A comparison of the product mixture from dry-down of tg alone with that resulting from dry-down of tg with Ala following separation via hydrophobicity-based C18-HPLC allowed identification of various polymers that contain Ala (Fig. 2c). We confirmed the identity of one of the observed products that is composed of a terminal tg linked with an amide to Ala, herein termed $\operatorname{tg} \mathrm{A}$, by comparison to a pure synthesized $\operatorname{tg} \mathrm{A}$ standard (Supplementary Fig. 5). In addition, one of the $\operatorname{tgAA}$ oligomers was also identified using a synthesized standard with the sequence of a terminal tg linked through two consecutive amide bonds to Ala residues (Supplementary Fig. 6). Kinetic analysis shows gradual oligomer formation by tg and Ala for up to seven days in the dry state (Supplementary Fig. 7).

To confirm amide bond formation during drying of amino acids with mercaptoacids, we performed direct infusion electrospray high-resolution tandem MS (MS/MS) analysis of tg and Ala drydown reaction products. The mixtures were resolved by C18-HPLC, and isolated peaks were collected for analysis. As mentioned above, identification of the tgA product peak was accomplished using an authentic standard, and the presence of an amide bond in this product was verified with MS/MS (Fig. 3). The tgA sequence was validated by the small mass error, $-1.3 \mathrm{mDa}$, the MS/MS mercaptopeptide fingerprint, water and $\mathrm{CO}_{2}$ neutral losses and $y_{1}$ ion detection for the Ala residue in the $\mathrm{C}$-termini. Further structural validation was performed by comparing the $\operatorname{tg} \mathrm{A}$ MS/MS spectra with that of the $\operatorname{tg} \mathrm{A}$ authentic standard, which showed almost identical fragment ions (Supplementary Fig. 8a). Characterization of other peaks by MS/MS identified the formation of additional amide bonds in other sequences (Supplementary Fig. 8b). For example, fragment ions observed for $\mathrm{m} / \mathrm{z} 233.1 \mathrm{MS} / \mathrm{MS}$ analysis correspond to the sequence $\operatorname{tgAA}$ (Supplementary Fig. 8b). 


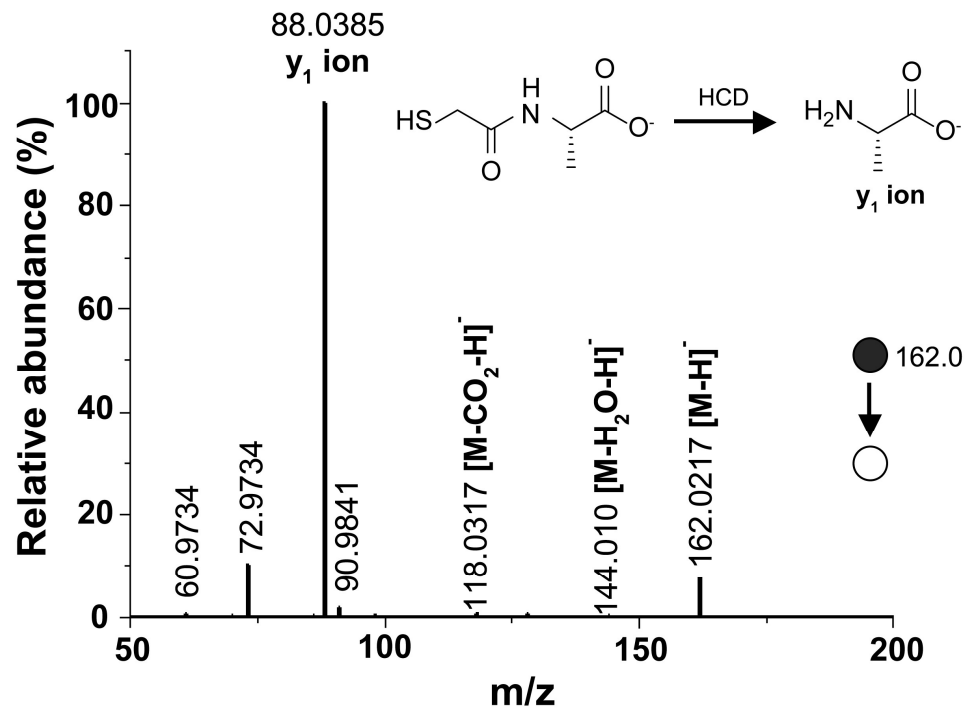

Fig. 3. Evidence for the formation of amide bonds upon dry-down of an amino acid and a mercaptoacid via MS/MS Higher-energy C-trap dissociation (HCD) analysis. tg and Ala were dried at $65{ }^{\circ} \mathrm{C}$ for seven days; the resulting products were analyzed by negative-ion mode MS/MS. Tandem MS analysis was conducted using $\mathrm{m} / \mathrm{z} 162.0$ as the precursor ion. Negative ion mode MS/MS analysis of the [tg+Ala-H2O] molecule showed a base peak at $\mathrm{m} / z$ 88.0385, being the $y 1$ ion of the sequence $\operatorname{tg} \mathrm{A}$. This fragmentation pattern is supportive of the formation of amide bonds in these dry-down reactions.

NMR characterization of product mixtures using synthesized standards. NMR characterization of dry-down reaction products support robust conversion of the starting building blocks into oligomers. The reaction of tg with Ala resulted in a relatively simple set of chemical envelopes in ${ }^{1} \mathrm{H}$ NMR spectra (Fig. 4 and Supplementary Figs. 9-15) that were interpreted by comparison to spectra of the pre-dried mixture and the tgA authentic standard. In unreacted Ala, the $\alpha$-proton chemical shift is centered at 3.95 ppm (Fig. 4a). After dry-down of Ala with tg, the intensity of this 3.95 ppm envelope decreases, and three envelopes of product $\alpha$-protons were observed, centered at 4.18, 4.35, and $4.52 \mathrm{ppm}$. The identities of these resonances as $\alpha$-protons of reacted Ala species were further supported by ${ }^{1} \mathrm{H}-{ }^{1} \mathrm{H}$ COSY NMR (Supplementary Fig. 11). 77\% of the $\alpha$-protons of Ala shifted down-field to $4.35 \mathrm{ppm}$, in accordance with the chemical shift of the $\alpha$-proton in the tgA standard (Fig. $\mathbf{4 b - c )}$. Notably, in the dried mixture there are many overlapping peaks in the 4.35 ppm envelope (Supplementary Fig. 13), which belong to reacted Ala species that have been amidated. Among the other product $\alpha$-protons in this envelope are those that belong to the tgAA sequence, as confirmed by the tgAA standard (Supplementary Figs. 14-15). Approximately 3\% of the $\alpha$-protons of Ala shifted down-field to $4.18 \mathrm{ppm}$, and additional $3 \%$ are located at the $4.52 \mathrm{ppm}$ region after the dry-down reaction. Integration of the free, un-reacted $\alpha$-proton resonance of Ala indicated that $83 \%$ of Ala was converted into oligomers after 1 week at $65{ }^{\circ} \mathrm{C}$. 


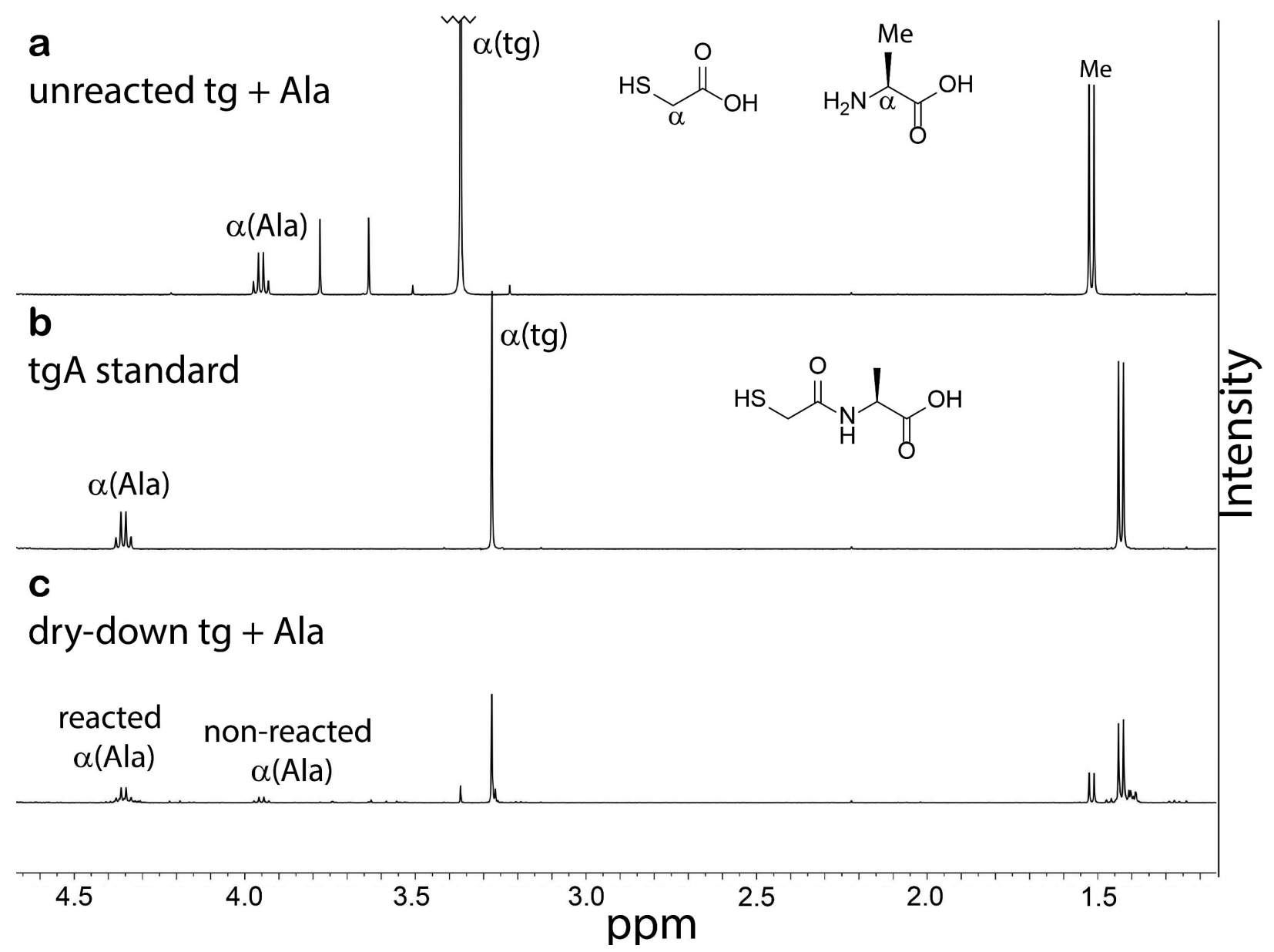

Fig. 4. Dry-down reactions of tg and Ala produce oligomers containing amide bonds. ${ }^{1} \mathrm{H}-\mathrm{NMR}$ spectra of: (a) A fresh, unreacted mixture of tg with Ala. (b) The authentic synthetic standard of Ala acylated with $\operatorname{tg}$ at the $\alpha$-amine $(\operatorname{tg} \mathrm{A})$ demonstrating the down-field shift of the $\alpha$-resonance upon amidation of Ala. (c) Products resulting from tg dried with Ala in a 5:1 molar ratio at $65^{\circ} \mathrm{C}$ for seven days.

\section{Robust formation of proto-peptides under a wide range of $\mathrm{pH}$ and temperature conditions.}

To investigate the generality of thiodepsipeptide formation via drying mercaptoacids with amino acids, we combined either tg or thiolactic acid (ta) with various amino acids, including glycine (Gly), Ala, phenylalanine (Phe), or cysteine (Cys). Analysis of these dry-down reactions via both MS and HPLC confirmed the formation of complex product mixtures that were consistent with oligomer formation (Supplementary Figs. 16-25). As expected, dry-down of the amino acids in the absence of mercaptoacids did not produce oligomers (Supplementary Figs. 17, 19, 21, and 23).

The reactions described above were carried out using unbuffered, mildly acidic conditions ( $\mathrm{pH} \sim 3$ ). Based on the strong nucleophilicity of the thiol moiety we postulated that thiodepsipeptides could form at $\mathrm{pH}>3$. To test this possibility, we carried out dry-down reactions containing mixtures of $\operatorname{tg}$ and Ala in which the initial reaction $\mathrm{pH}$ was adjusted to $3.5,5.5,6.5$, or 7.0. One, 5, 10, or 20 equivalents of imidazole were added with respect to the amount of amino acid to adjust the pH. HPLC and MS analyses indicated 
that thiodepsipeptide formation is most robust under acidic conditions. Nevertheless, thiodepsipeptide were still observed in good yields at every pH tested (Fig. 5a and Supplementary Fig. 26). Specifically, ${ }^{1} \mathrm{H}$ NMR analysis indicated that $90 \%$ of Ala was converted into oligomers upon dry-down with tg at $\mathrm{pH} 3.5,89 \%$ was converted at $\mathrm{pH} 5.5,71 \%$ at $\mathrm{pH} 6.5$, and $42 \%$ at $\mathrm{pH} 7.0$ (Supplementary Figs. 27-30).

While we initially focused on formation of thiodepsipeptides under evaporative dry-down conditions that shift the thermodynamic equilibrium towards condensation-dehydration products, we postulated that thiodepsipeptide formation might also be possible to some extent in water. Indeed, following incubation of a mixture of tg and Ala at $65{ }^{\circ} \mathrm{C}$ for one week in water with a closed reaction vial, various product peaks are evident, some of which contain amide bonds (Supplementary Fig. 31). As expected, fewer products are formed in the solution state compared to dry-down conditions (Supplementary Fig. 31).

Thiodepsipeptide formation is temperature-dependent and occurs under relatively mild temperatures, implying relatively low activation energies. Products are detected in dry-down reactions containing mixtures of tg and Ala at temperatures as low as $25^{\circ} \mathrm{C}(\mathbf{F i g}$. 5b). NMR analysis indicated that $4 \%$ of Ala converted into oligomers upon dry-down with tg at $25{ }^{\circ} \mathrm{C}$, with $6 \%$ converted at $37{ }^{\circ} \mathrm{C}, 23 \%$ at $50{ }^{\circ} \mathrm{C}$, and $83 \%$ at $65{ }^{\circ} \mathrm{C}$ after 1 week (Fig. $5 \mathbf{b}$ and Supplementary Figs. 32-34).
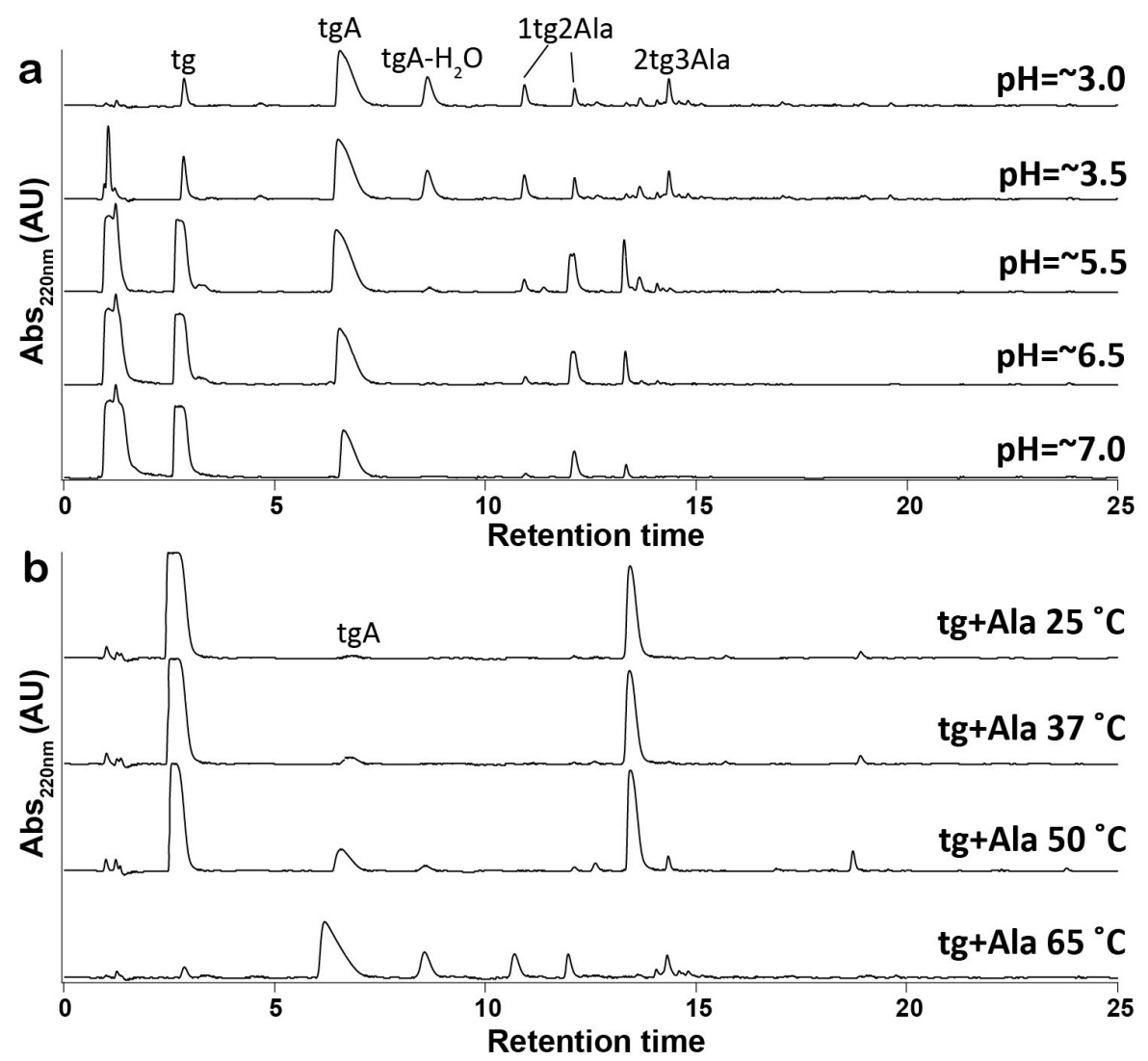
Fig. 5. Thiodepsipeptide formation occurs under a range of $\mathrm{pH}$ conditions and temperatures. (a) Chromatograms of products resulting from tg and Ala, in a 5:1 molar ratio, being dried at $65^{\circ} \mathrm{C}$ for 1 week in various initial reaction $\mathrm{pH}$. Products are observed throughout the whole range of $\mathrm{pH}$ conditions tested. (b) Chromatograms illustrating the temperature dependence of thiodepsipeptide formation under unbuffered, acidic conditions ( $\mathrm{pH} \sim 3$ ). Products are detected in dry-down reactions containing mixtures of tg and Ala even at $25^{\circ} \mathrm{C}$.

Comparison between thiodepsipeptide and depsipeptide formation. We directly compared the formation of thiodepsipeptides through dry-down reactions of mercaptoacids and amino acids to analogous reactions containing mixtures of hydroxy acids and amino acids, the latter resulting in the formation of depsipeptides ${ }^{29-31}$ Both depsipeptide and thiodepsipeptide polymerization occurs under acidic conditions, but in contrast to thiodepsipeptides, no depsipeptide products are formed at pH 7 (Supplementary Fig. 35). The activation energy for formation of thiodepsipeptides is lower than for depsipeptides. While products were detected in dry-down reactions containing mixtures of tg and Ala even at $25^{\circ} \mathrm{C} \mathrm{(Fig.} \mathrm{5b),} \mathrm{no} \mathrm{polymers}$ were observed under these conditions for dry-down reactions containing the equivalent hydroxy acid and amino acid mixture (Supplementary Fig. 36). In contrast to thiodepsipeptide formation, which proceeded to some extent in water (Supplementary Fig. 31), incubation of a mixture of glycolic acid (glc) (the hydroxy acid analog of tg) with Ala at $65{ }^{\circ} \mathrm{C}$ for one week did not produce oligomers (Supplementary Fig. 37). The extent of conversion of a given amino acid when dried-down with either mercaptoacids or hydroxy acids is the same. Roughly similar conversion of Ala into oligomers was evident in both systems, where $64 \%$ of Ala converted into oligomers with glc (Supplementary Fig. 38), compared to 83\% conversion with tg (Fig. 4).

\section{Discussion}

The reactions reported here were conducted in an anaerobic chamber to minimize oxidation of thiolcontaining compounds. This experimental setup of anaerobic conditions is plausibly prebiotic, mimicking the atmosphere of the prebiotic Earth, long before the great oxidation event that occurred only around 2 billion years ago ${ }^{39}$ Nonetheless, we carried out a test reaction involving a dry-down of tg with Ala under present-day oxic conditions and observed formation of various side-products, including the prevalence of products containing disulfide bonds (Supplementary Fig. 39). This result confirmed the importance of anaerobic conditions in experiments designed to explore the possibility for thioester-mediated peptide bond formation in an early Earth environment. Additionally, to examine the monomer exchange of thiodepsipeptides under a plausible geochemical scenario of fluctuating environmental conditions, we performed iterative dry-wet cycles. We anticipate net condensation-dehydration during the dry phases and net hydrolysis during the wet phases. The results show that thiodepsipeptides are formed under these 
conditions, albeit with lower yield compared to continuous dry-state conditions (Supplementary Fig. 40), as expected.

In conclusion, we have presented a plausible prebiotic scenario in which thiodepsipeptides are formed over a wide range of conditions: varying hydration levels, temperature, and $\mathrm{pH}$. Previously, short thiol-terminated compounds and thioesters have been investigated in both aqueous and non-aqueous solutions as building block units for dynamic combinatorial libraries. ${ }^{40-44}$ Based on the results presented here we propose that incorporation of even simple mercaptoacids into these systems might offer new avenues for dynamic combinatorial chemistry in water or under dry-down conditions. Of importance to origins research, mixtures of different building blocks, including amino acids, mercapto acids, and hydroxy acids are, arguably, more prebiotically plausible than pure amino acids as starting conditions for polymer evolution. Taken together, the combined results of our study suggest that thiodepsipeptides could have formed robustly on prebiotic Earth and participated in the early stages of chemical evolution that eventually gave rise to life.

\section{Methods}

\section{Standards}

Synthesis of standards is presented in the Supporting Information.

\section{Dry-down reactions}

All dry-down reactions that contained mercaptoacids were carried out in a Coy anoxic chamber $(97 \%$ Ar, $3 \% \mathrm{H}_{2}$ headspace) to minimize oxidation, unless stated otherwise.

For polymerization experiments, aqueous solutions of mercaptoacids and amino acids (typically $166.66 \mathrm{mM}$ amino acid and $833.33 \mathrm{mM}$ mercaptoacid) were allowed to dry in Eppendorf tubes at various temperatures in a dry block heater $\left(25^{\circ} \mathrm{C}, 37^{\circ} \mathrm{C}, 50^{\circ} \mathrm{C}\right.$, or $\left.65^{\circ} \mathrm{C}\right)$, with the lid open, for various durations. Prior to analysis, samples were resuspended with $90 \%$ ultrapure water, $10 \%$ acetonitrile to a given concentration, vortexed, and sonicated.

For polymerization experiments with imidazole added, an imidazole solution was aliquoted and lyophilized prior to addition of thioglycolic acid and alanine in the anaerobic chamber.

For polymerization experiments of control depsipeptides, aqueous solutions of glycolic acid and amino acids were allowed to dry in Eppendorf tubes at various temperatures in an oven or on a dry block $\left(25^{\circ} \mathrm{C}\right.$, $37^{\circ} \mathrm{C}, 50{ }^{\circ} \mathrm{C}, 65^{\circ} \mathrm{C}$, or $\left.85^{\circ} \mathrm{C}\right)$.

For dry-down experiments of thiodepsipeptides under oxic conditions, thioglycolic acid and L-Ala were allowed to dry in an oven at $65^{\circ} \mathrm{C}$ for seven days.

For the pH-dependent dry-down experiment, samples were dried under different $\mathrm{pH}$ conditions using addition of different number of equivalents of imidazole. For the $\mathrm{pH}$-dependent experiment in solution, 
samples were incubated in water $(\mathrm{pH} 3.0,55.55 \mathrm{mM}$ Ala and $277.75 \mathrm{mM} \mathrm{tg})$ or in the presence of 20eq $\mathrm{Na}_{2} \mathrm{HPO}_{4}(\mathrm{pH} \sim 7.0$, referring to the amount of the amino acid, $38.46 \mathrm{mM}$ Ala and $192.30 \mathrm{mM} \mathrm{tg}$ ).

For dry-wet cycling experiment, aqueous solutions of mercaptoacids and amino acids were allowed to dry in Eppendorf tubes at $65^{\circ} \mathrm{C}$ in a dry block, with the cap open. After 24 hours samples were cooled back to room temperature, resuspended with $200 \mu \mathrm{L}$ of $\mathrm{H}_{2} \mathrm{O}$, mixed and were allowed to dry with an open cap. This was repeated five more times for a total reaction time of 1 week.

\section{Direct Infusion Mass spectrometry}

All peak assignments correspond to $[\mathrm{M}-\mathrm{H}]^{-}$ions, unless otherwise noted. Samples were directly infused into a mass spectrometer using the parameters: solvent: $95 \% \mathrm{H}_{2} \mathrm{O}, 5 \%$ acetonitrile. Flow rate: $0.5 \mathrm{~mL} / \mathrm{min}$. Five $\mu \mathrm{L}$ injection with $\mathrm{H}_{2} \mathrm{O}$ needle wash. UV detection at 210, 257 or $280 \mathrm{~nm} .0 .6 \mathrm{~cm}$ path length. Scanning $\pm 65- \pm 2000 \mathrm{~m} / z$. Equipment: ESI-MS - Agilent 6130 single quadrupole MS (Agilent Technologies, Santa Clara, CA) with UV detector coupled to Agilent 1260 HPLC. Capillary voltage: 2.0kV. Fragmentor voltage: $70 \mathrm{~V}$.

Reactivity of Ala in dry-down reactions was determined by integration of the free, un-reacted $\alpha$-proton, normalized to the integration of the methylene protons of Ala.

\section{High-resolution MS and MS/MS analysis}

For direct infusion high-resolution analysis, the fractions collected for different important chromatographic regions were diluted 1 to 10 in a 1:1 acetonitrile-water solution. Tandem MS analyses were performed using a Q-Exactive hybrid quadrupole-Orbitrap mass spectrometer (Thermo Scientific, San Jose, CA) operating with a heated electrospray interface (HESI). Analyses of products from dry-down reactions were carried out in negative ion mode with the following operating conditions: maximum ion injection time (50 ms), FT resolution (17,500), AGC target (1e6), HCD energy (NCE = 35), $\mathrm{MS}^{2}$ isolation width $m / z= \pm 1.5$, voltage $2.8 \mathrm{kV}$, capillary temperature $\left(320^{\circ} \mathrm{C}\right)$, sheath gas $(10 \mathrm{~L} / \mathrm{min})$, auxiliary gas $(2 \mathrm{~L} / \mathrm{min})$, S-lens RF level 50 .

The Q-Exactive mass spectrometer was calibrated using Pierce ${ }^{\mathrm{TM}}$ Negative Ion Calibration Solution (Thermo Scientific, San Jose, CA). Data were processed using Xcalibur ${ }^{\mathrm{TM}}$ version 4.0.

\section{High Performance Liquid Chromatography}

HPLC analyses were conducted using an Agilent 1260 quaternary pump and Agilent 1260 Autosampler with DAD UV-vis detector, with a path length of $1.0 \mathrm{~cm}$. Samples were separated using a Phenomenex Kinetex 2.6mmxB-C18100 , LC column $150 \times 2.1 \mathrm{~mm}$. Column temp: $25^{\circ} \mathrm{C}$. $10 \mu \mathrm{L}$ Injection. Solvents: Solvent gradient was: A) $0.1 \%$ formic acid in LC-MS grade water, B) LC-MS grade acetonitrile. Flow Rate: $0.3 \mathrm{~mL} / \mathrm{min}$. Gradient: $5 \mathrm{~min} 100 \% \mathrm{~A}, 0 \% \mathrm{~B} ; 20 \mathrm{~min} \operatorname{ramp}$ to $45 \% \mathrm{~A}, 55 \% \mathrm{~B} ; 10 \mathrm{~min} 0 \% \mathrm{~A}, 100 \% \mathrm{~B} ; 1$ $\min \operatorname{ramp} 100 \% \mathrm{~A}, 0 \% \mathrm{~B} ; 14 \min 100 \% \mathrm{~A}, 0 \% \mathrm{~B}$. Wavelengths monitored: 210 and $220 \mathrm{~nm}$, with entire spectrum 180-400 nm collected in $2 \mathrm{~nm}$ steps.

\section{Liquid Chromatography-Mass Spectrometry}


HPLC equipment and chromatography conditions: Agilent 1290 HPLC pump and thermostat. Agilent 1260 Autosampler and DAD UV-vis detector with path length of $0.6 \mathrm{~cm}$. Agilent 1260 quaternary pump and RID. Column: Phenomenex Kinetex 2.6 mmxB-C $18100 \AA$, LC column 150x2.1 mm. H15-191145, 5603145. Column temp: $25^{\circ} \mathrm{C}$. Ten $\mu \mathrm{L}$ Injection with needle wash, $100 \mu \mathrm{L} / \mathrm{s}$ injection speed. Solvents: A) $0.1 \%$ formic acid in LC-MS grade water, B) LC-MS grade acetonitrile. Flow rate: $0.3 \mathrm{~mL} / \mathrm{min}$. Gradient: $5 \mathrm{~min}$ $100 \%$ A, $0 \%$ B; 20 min ramp to $45 \%$ A, 55\% B; 10 min 0\% A, 100\% B; 1 min ramp 100\% A, 0\% B; 9 min $100 \%$ A, $0 \%$ B. Wavelengths recorded: 210 and $220 \mathrm{~nm}$, with entire spectrum 180-400 nm detected in 2 $\mathrm{nm}$ steps. This system was coupled to an Agilent 6130 single quadrupole mass spectrometer using the following parameters: Scanning $\pm 65- \pm 2000 \mathrm{~m} / z$. Capillary voltage: $2.0 \mathrm{kV}$. Fragmentor voltage: $70 \mathrm{~V}$. Single ion monitoring was carried out using the same system and tracking particular ions (as listed in Figure S32).

\section{NMR spectroscopy}

Samples were dissolved in a phosphate buffer ( $\mathrm{pH}$ 2.5) in $\mathrm{D}_{2} \mathrm{O}$ and ${ }^{1} \mathrm{H}-\mathrm{NMR}$ spectra were recorded using Bruker Avance III-HD-500 MHz spectrometer. Temperature was $298 \mathrm{~K}$, using a t1 relaxation delay of 15 S, collecting 64 scans. All spectra were processed and plotted using the MestReNova Software. Overall conversion of alanine monomer into polymers was estimated from integration of the free, nonamidated $\alpha$ proton ${ }^{1} \mathrm{H}$ NMR resonance.

\section{IR spectroscopy}

IR data was obtained on a Thermo Nicolet 4700 FTIR spectrometer. Prior to analysis, samples (10 $\mu 1,50$ $\mathrm{mM}$ amino acid or $250 \mathrm{mM}$ mercaptoacid monomer) were placed on Durapore ${ }^{\circledR}$ hydrophobic PVDF membranes with a pore size of $0.22 \mu \mathrm{m}$ (\#GVHP04700, Millipore Sigma) and allowed to dry. Dried samples were analyzed in an Attenuated Total Reflectance (ATR) sample chamber. Spectra were backgroundsubtracted from 400 to $4000 \mathrm{~cm}^{-1}$ and signal-averaged (16 scans per spectrum).

\section{Data Availability}

All the data supporting the findings of this study are available within the main text and its Supplementary Information. Data are also available from the corresponding authors upon request.

\section{Acknowledgments}

We acknowledge Drs. Arthur L. Weber, Ramanarayanan Krishnamurthy, Jay Forsythe, and David Fialho for fruitful discussions. This research was supported by the NSF and the NASA Astrobiology Program under the NSF Center for Chemical Evolution [CHE-1504217]. 


\section{References}

Frenkel-Pinter, M., Samanta, M., Ashkenasy, G. \& Leman, L. J. Prebiotic Peptides: Molecular Hubs in the Origin of Life. Chem. Rev. (2020).

Huber, C. \& Wächtershäuser, G. Activated acetic acid by carbon fixation on ( $\mathrm{Fe}, \mathrm{Ni}) \mathrm{S}$ under primordial conditions. Science 276, 245-247 (1997).

Hartman, H. \& Smith, T. F. Origin of the genetic code is found at the transition between a thioester world of peptides and the phosphoester world of polynucleotides. Life 9, 69 (2019). Chandru, K., Gilbert, A., Butch, C., Aono, M. \& Cleaves, H. J. The abiotic chemistry of thiolated acetate derivatives and the origin of life. Sci. Rep. 6, 29883 (2016).

Wächtershäuser, G. Groundworks for an evolutionary biochemistry: the iron-sulphur world. Prog. Biophys. Mol. Biol. 58, 85-201 (1992).

Wächtershäuser, G. Evolution of the first metabolic cycles. Proc. Natl. Acad. Sci. USA 87, 200-204 (1990).

Kricheldorf, H. R. \& Schwarz, G. Poly (thioester) s. J. Macromol. Sci., Pure Appl. Chem. 44, 625649 (2007).

Cecil, R. \& McPhee, J. in Advances in Protein Chemistry Vol. 14 255-389 (Elsevier, 1959).

Russell, M. J. \& Martin, W. The rocky roots of the acetyl-CoA pathway. Trends Biochem. Sci. 29, 358-363 (2004).

Keefe, A. D., Newton, G. L. \& Miller, S. L. A possible prebiotic synthesis of pantetheine, a precursor to coenzyme A. Nature 373, 683-685 (1995).

1 Blöchl, E., Keller, M., Wachtershäuser, G. \& Stetter, K. O. Reactions depending on iron sulfide and linking geochemistry with biochemistry. Proc. Natl. Acad. Sci. USA 89, 8117-8120 (1992).

2 Chandru, K., Jia, T. Z., Mamajanov, I., Bapat, N. \& Cleaves, H. J. Prebiotic oligomerization and self-assembly of structurally diverse xenobiological monomers. Sci. Rep. 10, 1-14 (2020).

Nanda, J. et al. Emergence of native peptide sequences in prebiotic replication networks. Nat. Commun. 8, 1-9 (2017).

Mukherjee, R., Cohen-Luria, R., Wagner, N. \& Ashkenasy, G. A Bistable Switch in Dynamic Thiodepsipeptide Folding and Template-Directed Ligation. Angew. Chem. 127, 12629-12633 (2015).

5 Foden, C. S. et al. Prebiotic synthesis of cysteine peptides that catalyze peptide ligation in neutral water. Science 370, 865-869 (2020).

6 Sanden, S. A., Yi, R., Hara, M. \& McGlynn, S. E. Simultaneous synthesis of thioesters and ironsulfur clusters in water: two universal components of energy metabolism. Chem. Commun. 56, 11989-11992 (2020).

Wu, L.-F. \& Sutherland, J. D. Provisioning the origin and early evolution of life. Emerging topics in life sciences 3, 459-468 (2019).

De Duve, C. The beginnings of life on earth. Am. Sci. 83, 428-437 (1995). Heinen, W. \& Lauwers, A. M. Organic sulfur compounds resulting from the interaction of iron sulfide, hydrogen sulfide and carbon dioxide in an anaerobic aqueous environment. Orig. Life Evol. Biosph. 26, 131-150 (1996).

Wieland, T., Bokelmann, E., Bauer, L., Lang, H. \& Lau, H. Über Peptidsynthesen. 8. Mitteilung Bildung von S-haltigen Peptiden durch intramolekulare Wanderung von Aminoacylresten. Liebigs Ann. Chem. 583, 129-149 (1953).

1 Weber, A. L. \& Orgel, L. E. The formation of peptides from glycine thioesters. J. Mol. Evol. 13, 193-202 (1979).

Weber, A. L. Prebiotic formation of 'energy-rich'thioesters from glyceraldehyde and Nacetylcysteine. Orig. Life Evol. Biosph. 15, 17-27 (1984). 
Weber, A. L. Prebiotic amino acid thioester synthesis: thiol-dependent amino acid synthesis from formose substrates (formaldehyde and glycolaldehyde) and ammonia. Orig. Life Evol. Biosph. 28, 259-270 (1998).

24 Weber, A. L. Aqueous synthesis of peptide thioesters from amino acids and a thiol using 1, 1'carbonyldiimidazole. Orig. Life Evol. Biosph. 35, 421-427 (2005).

25 Liu, R. \& Orgel, L. E. Oxidative acylation using thioacids. Nature 389, 52-54 (1997).

26 Okamoto, R. et al. Regioselective $\alpha$-peptide bond formation through the oxidation of amino thioacids. Biochemistry 58, 1672-1678 (2019).

27 Canavelli, P., Islam, S. \& Powner, M. W. Peptide ligation by chemoselective aminonitrile coupling in water. Nature 571, 546-549 (2019).

28 Keller, M., Blöchl, E., Wächtershäuser, G. \& Stetter, K. Formation of amide bonds without a condensation agent and implications for origin of life. Nature 368, 836-838 (1994).

29 Forsythe, J. G. et al. Ester-mediated amide bond formation driven by wet-dry cycles: A possible path to polypeptides on the prebiotic Earth. Angew. Chem. Int. Ed. 54, 9871-9875 (2015).

$30 \mathrm{Yu}, \mathrm{S} .-\mathrm{S}$. et al. Kinetics of prebiotic depsipeptide formation from the ester-amide exchange reaction. Phys. Chem. Chem. Phys. 18, 28441-28450 (2016).

31 Frenkel-Pinter, M. et al. Selective incorporation of proteinaceous over nonproteinaceous cationic amino acids in model prebiotic oligomerization reactions. Proc. Natl. Acad. Sci. USA 116, 16338-16346 (2019).

32 Wells, P. Linear Free Energy Relationships. Chem. Rev. 63, 171-219 (1963).

33 Rozenberg, M. \& Shoham, G. FTIR spectra of solid poly-l-lysine in the stretching NH mode range. Biophys. Chem. 125, 166-171 (2007).

34 Tewari, K. \& Vishnoi, N. A textbook of organic chemistry. (Vikas Publishing House, 1976).

$35 \mathrm{Kim}, \mathrm{E}$. et al. Synthesis of gold nanorod-embedded polymeric nanoparticles by a nanoprecipitation method for use as photothermal agents. Nanotechnology 20, 365602 (2009).

36 Montero-Rama, M. P., Liras, M., García, O. \& Quijada-Garrido, I. Thermo-and pH-sensitive hydrogels functionalized with thiol groups. Eur. Polym. J. 63, 37-44 (2015).

37 McEvoy, J. P. Characterizing carbonyls with Infrared spectroscopy: an introductory chemistry experiment in a molecular bioscience program. J. Chem. Educ. 91, 726-729 (2014).

38 Attar, A. R., Blumling, D. E. \& Knappenberger Jr, K. L. Photodissociation of thioglycolic acid studied by femtosecond time-resolved transient absorption spectroscopy. J. Chem. Phys. 134, 024514 (2011).

39 Okafor, C. D. et al. Iron mediates catalysis of nucleic acid processing enzymes: support for Fe (II) as a cofactor before the great oxidation event. Nucleic Acids Res. 45, 3634-3642 (2017).

40 Ura, Y. et al. Dynamic polythioesters via ring-opening polymerization of 1, 4-thiazine-2, 5-diones. Organic \& biomolecular chemistry 7, 2878-2884 (2009).

41 Ghosh, S. et al. Dynamic cyclic thiodepsipeptide libraries from thiol-thioester exchange. Org. Lett. 12, 1860-1863 (2010).

42 Dadon, Z., Samiappan, M., Shahar, A., Zarivach, R. \& Ashkenasy, G. A High-Resolution Structure that Provides Insight into Coiled-Coil Thiodepsipeptide Dynamic Chemistry. Angew. Chem. Int. Ed. 52, 9944-9947 (2013).

43 Carnall, J. M. et al. Mechanosensitive self-replication driven by self-organization. Science 327, 1502-1506 (2010).

44 Altay, Y., Tezcan, M. \& Otto, S. Emergence of a new self-replicator from a dynamic combinatorial library requires a specific pre-existing replicator. J. Am. Chem. Soc. 139, 13612-13615 (2017). 


\section{Graphical Abstract}
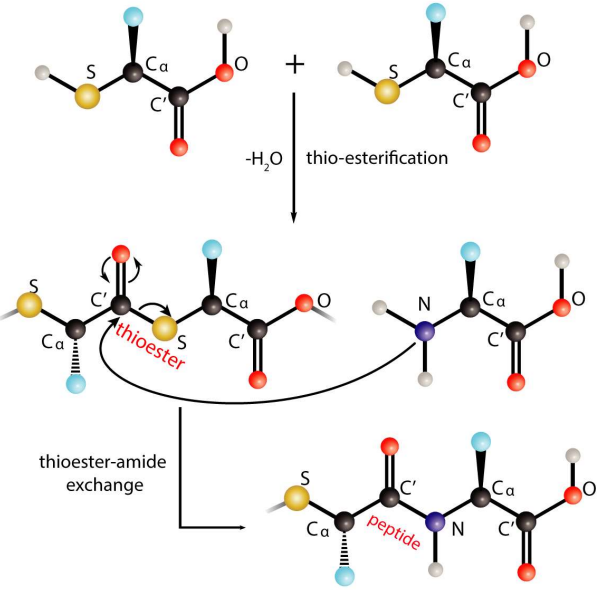